\title{
High Resolution Multiphoton Spectroscopy by a Tunable Free-Electron-Laser Light
}

\author{
M. Žitnik, ${ }^{1,2}$ A. Mihelič, ${ }^{1}$ K. Bučar, ${ }^{1}$ M. Kavčič, ${ }^{1}$ J.-E. Rubensson, ${ }^{3}$ M. Svanquist, ${ }^{3}$ J. Söderström, ${ }^{3}$ R. Feifel, ${ }^{3,4}$ C. Såthe, \\ Y. Ovcharenko, ${ }^{6}$ V. Lyamayev, ${ }^{7}$ T. Mazza, ${ }^{8}$ M. Meyer, ${ }^{8}$ M. Simon, ${ }^{9,10}$ L. Journel, ${ }^{9,10}$ J. Lüning, ${ }^{9,10}$ O. Plekan, ${ }^{11}$ M. Coreno, ${ }^{11}$ \\ M. Devetta, ${ }^{11}$ M. Di Fraia, ${ }^{12}$ P. Finetti, ${ }^{11}$ R. Richter, ${ }^{11}$ C. Grazioli, ${ }^{11}$ K. C. Prince, ${ }^{11,13}$ and C. Callegari ${ }^{11}$ \\ ${ }^{1}$ Jožef Stefan Institute, Jamova cesta 39, SI-1000 Ljubljana, Slovenia \\ ${ }^{2}$ Faculty of Mathematics and Physics, University of Ljubljana, Jadranska 19, SI-1000 Ljubljana, Slovenia \\ ${ }^{3}$ Uppsala University, Department of Physics and Astronomy, P.O. Box 530, S-75121 Uppsala, Sweden \\ ${ }^{4}$ University of Gothenburg, Department of Physics, SE-412 96 Gothenburg, Sweden \\ ${ }^{5}$ MAX IV Laboratory, Lund University, P.O. Box 118, 22100 Lund, Sweden \\ ${ }^{6}$ Technische Universität Berlin, Institut für Optik und Atomare Physik, Eugene-Wigner-Building Hardenbergstrasse 36, \\ D-10623 Berlin, Germany \\ ${ }^{7}$ Institute of Physics, University of Freiburg, Hermann-Herder Strasse 3a, D-79104 Freiburg, Germany \\ ${ }^{8}$ European XFEL GmbH, Albert-Einstein-Ring 19, 22761 Hamburg, Germany \\ ${ }^{9}$ Sorbonne Université, UPMC Univ Paris 06, UMR 7614, LCPMR, 75005 Paris, France \\ ${ }^{10}$ CNRS, UMR 7614, LCPMR, 75005 Paris, France \\ ${ }^{11}$ Elettra-Sincrotrone Trieste, Strada Statale 14-km 163, 5, I-34149 Basovizza, Trieste, Italy \\ ${ }^{12}$ Department of Physics, University of Trieste, Via Valerio 2, I-34124 Trieste, Italy \\ ${ }^{13}$ eChemistry Laboratory, Faculty of Life and Social Sciences, Swinburne University of Technology, \\ Hawthorn, Melbourne, Victoria 3122, Australia
}

(Received 22 April 2014; published 7 November 2014)

\begin{abstract}
Seeded free electron lasers theoretically have the intensity, tunability, and resolution required for multiphoton spectroscopy of atomic and molecular species. Using the seeded free electron laser FERMI and a novel detection scheme, we have revealed the two-photon excitation spectra of dipole-forbidden doubly excited states in helium. The spectral profiles of the lowest $(-1,0)^{+1}{ }^{1} S^{e}$ and $(0,1)^{0}{ }^{1} D^{e}$ resonances display energy shifts in the meV range that depend on the pulse intensity. The results are explained by an effective two-level model based on calculated Rabi frequencies and decay rates.
\end{abstract}

DOI: 10.1103/PhysRevLett.113.193201

PACS numbers: $34.50 . \mathrm{Fa}, 41.60 . \mathrm{Cr}$

Free-electron lasers (FELs) are excellent light sources for multiphoton single-color target excitation with photon energy in the extreme ultraviolet (EUV) range, and the only sources available in the $\mathrm{x}$-ray energy range. High harmonic generation sources have been used in the EUV range [1,2], but they generally lack the broad tunability and intensity of FELs. Most FELs operate on the basis of self-amplified spontaneous emission and display relatively large shot-byshot fluctuations in wavelength (and intensity) [3-6]. While this does not prevent the accumulation of spectrally sorted signals, such sources are quite inefficient when energy scans are required over wavelength intervals much smaller than the fluctuation range $(\gtrsim 1 \%)$, or when the spectral features of the target are much narrower than the available single-shot (single spike) resolution $(\gtrsim 0.2 \mathrm{eV})$. The FERMI seeded free-electron-laser emits in the EUV energy region with a narrow $(\sim 0.03 \mathrm{eV})$ and stable $(\sim 0.1 \%)$ bandwidth, stable intensity, and is easily tuned over wide or narrow wavelength intervals [7].

In atoms and molecules there is a class of short-lived resonances, the so-called superexcited states with excitation energies above the first ionization threshold and spectral widths in the meV range [8-14]. These correlated, doubly excited electronic states are coupled to the continuum, and accurate description of resonance excitation and decay dynamics requires state-of-the-art modeling. In the vicinity of superexcited states, the decay branching probabilities are strongly modified and specific decay channels are opened. The dipole-allowed subset of resonances can be excited by synchrotron light and has been extensively studied over the years [15-18], improving our understanding of many-body interactions in these systems. However, the dipole-forbidden states have been less explored [19,20].

High intensity and improved resolution of the new seeded FEL sources enable targeted multiphoton excitation of resonances beyond the limitation of the dipole selection rule. To maintain high resolution in such experiments, the resolving power of the detector must at least match that of the probe. This represents a difficult task when the nonlinear preparation of superexcited states requires photon energy above the first ionization threshold-in this case the ions and fast electrons emitted in the resonance decay are swamped by ions and electrons from the onephoton ionization. Under such experimental conditions, the inherent spectroscopic conflict between resolution and efficiency is still not resolved in a satisfactory manner; i.e., the existing electron spectrometers are either efficient, but with low resolving power $(<100$, [21,22]), or not 
sufficiently efficient to separate the weak resonant signal from the background noise (acceptance solid angle $\lesssim 10^{-2}$ ).

By detection of neutral metastable (MS) fragments which are produced exclusively by the fluorescence decay or neutral dissociation of superexcited states, the spectrometer can be bypassed to make spectroscopy dependent on FEL resolution only. The MS states are long lived either because the fragments are highly excited, or because decay of the MS states to the ground state violates the dipole selection rule [23]. The MS species can be easily discriminated against charged particles and they can carry enough internal energy to trigger the microchannel-plate detector. In experiments with collimated atomic beams the MS atoms can be efficiently intercepted by the detector away from the target region resulting in a practically background-free signal.

We have set up a detection of MS decay products to study multiphoton excitation of resonances with a new tunable high resolution free-electron laser source. The method is tested on the fundamental process of two-photon excitation to He doubly excited states (DES) with excitation energies surpassing twice the ionization threshold [24]. The results evidence a direct two-photon absorption path to the DES below the $N=2$ ionization threshold [Fig. 1(a)] which has not been reported before and validate previous theoretical studies of the two-photon ionization cross section [25-27]. The DES are the prototype of correlated atomic states and ${ }^{2 S+1} L^{\pi}$ series with selected spin, orbital angular momentum and parity are grouped according to approximate correlation quantum numbers $(K, T)^{A}[8,28]$. The DES correlation types can be abbreviated by letters [8]: for the ${ }^{1} S^{e}$ and ${ }^{1} D^{e}$ states converging to the $N=2$ threshold, that are in the focus of this work, $n a$ is equivalent to $n(1,0)^{+}$, whereas $n b$ stands for $n(-1,0)^{+}$and $n(0,1)^{0}$ quantum numbers of states associated with the ${ }^{1} S^{e}$ and ${ }^{1} D^{e}$ symmetry, respectively.

In the last decade, the fluorescence decay channel [29], with branching to the $1 s 2 s$ metastable states [30], was proven a sensitive tool for studying DES with high resolution synchrotron sources in the field-free [31-34], and static electric [20,35,36] and magnetic [37] field environment.
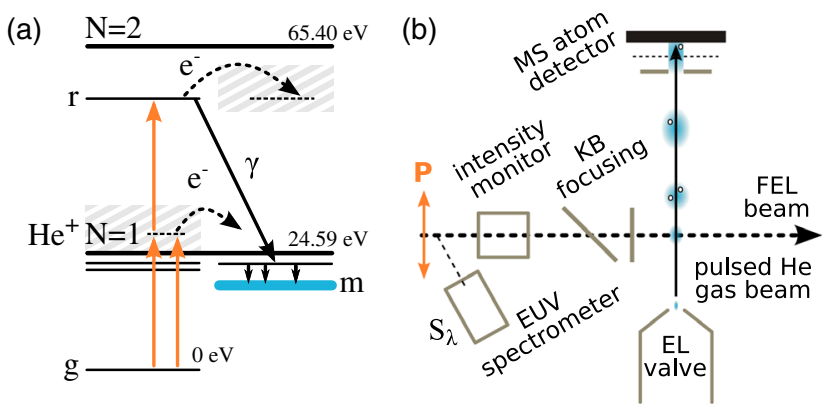

FIG. 1 (color online). (a) Transition diagram for the two-photon excitation to the resonance state and its decay: $g$, the ground state; $r$, the resonance state; $m$, the metastable states (see main text for description). (b) Experimental scheme.
At the same time, following the advent of intense EUV light sources, several benchmark experiments with ions and electrons were performed to study the response of the $\mathrm{He}$ atom to the intense laser fields [38-43]. This work brings together both research lines to perform a high resolution two-photon spectroscopy on DES.

The experiment was performed at the low density matter (LDM) beamline of FEL facility FERMI, Italy, operating in horizontal polarization at the sixth harmonic of the seed laser. An increase of the MS atom yield was detected when the FEL photon energy was swept across the energy region corresponding to half the excitation energy of the low lying DES. The FERMI diagnostics [44] provided the spectrum $\left(S_{\lambda}\right)$ and the total energy $(W)$ of each light pulse in the target region [Fig. 1(b)]. The LDM end station has been described elsewhere [45]. Two main components used in this study were the pulsed Even-Lavie valve and the MS atom detector. As seen in Fig. 2, at the maximum pulse energy, the highest detected MS yield was about 4 counts/pulse at the top of the $3 b{ }^{1} D^{e}$ resonance. A complete set of experimental data is presented in Fig. 3.

To understand the underlying processes, we have devised an effective two-level model with an explicit branching of the upper state to the MS states [Fig. 1(a)]. The time evolution of the system is described by the following set of equations:

$$
\begin{aligned}
& \dot{\rho}_{g g}=-\Gamma_{g} \rho_{g g}-i \frac{\Omega_{g r}}{2} \rho_{r g}+i \frac{\Omega_{g r}^{*}}{2} \rho_{r g}^{*}, \\
& \dot{\rho}_{r r}=-\Gamma_{r} \rho_{r r}-i \frac{\Omega_{r g}}{2} \rho_{r g}^{*}+i \frac{\Omega_{r g}^{*}}{2} \rho_{r g},
\end{aligned}
$$

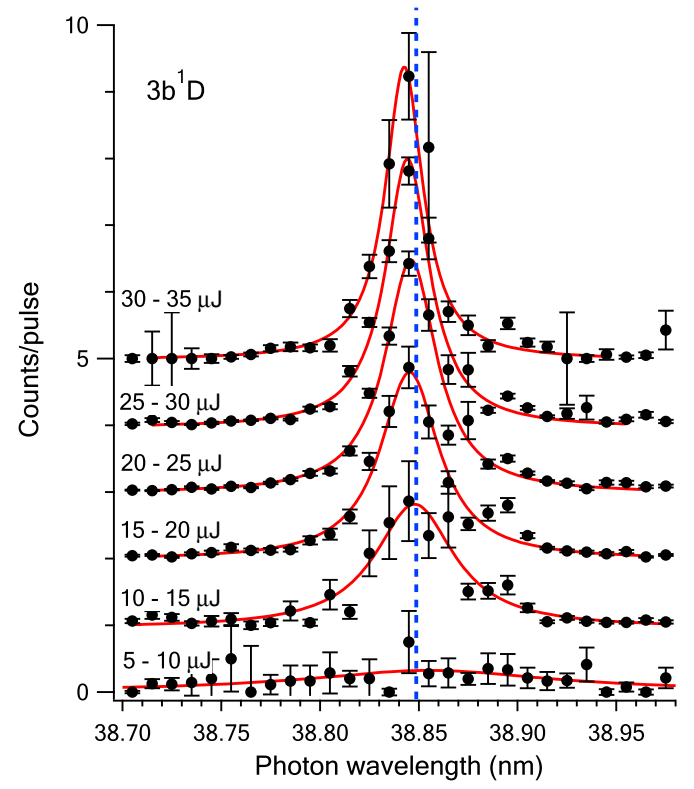

FIG. 2 (color online). The metastable atom yield measured in the wavelength region of the $3 b^{1} D^{e}$ resonance at different pulse energies $W$ (circles). The solid (red) lines show the least-squares (Lorentzian) fit. The individual spectra are shifted vertically for clarity. 


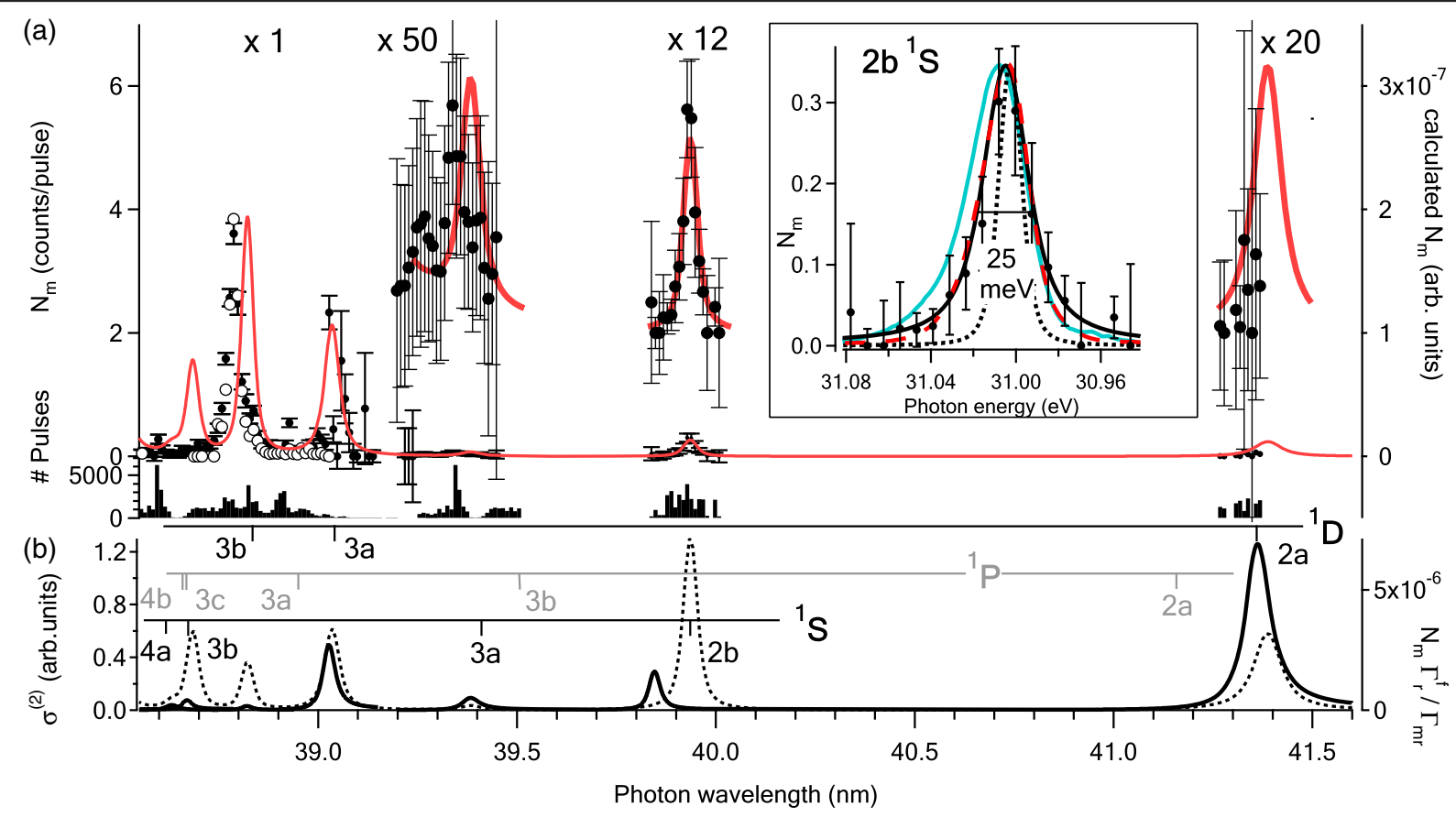

FIG. 3 (color online). (a) The measured MS yield (full circles) at saturation compared to the model result [gray (red) line]. Empty circles correspond to the data obtained by a more restrictive pulse filtering [46]. The histogram of FEL pulses after the filtering. Inset: The measured MS yield of the $2 b^{1} S^{e}$ state collected at $30 \mu \mathrm{J} \leq W \leq 60 \mu \mathrm{J}$ compared to the typical EUV pulse spectrum $S_{\lambda}$ [gray (cyan) line], to its autoconvolution $(S * S)_{2 \lambda}$ [dashed red (gray) line], and to the two model curves: with $\left(\gamma_{L}=16\right.$ meV, black line) and without an extra line broadening $\left(\gamma_{L}=0\right.$, dotted black line). (b) Theoretical two-photon ionization cross section from Ref. [25], broadened by the experimental resolution (full line) and the calculated total fluorescence yield spectrum (dotted line).

$$
\begin{gathered}
\dot{\rho}_{r g}=\left(i \Delta-\frac{\Gamma_{g}}{2}-\frac{\Gamma_{r}}{2}-\gamma_{L}\right) \rho_{r g}-i \frac{\Omega_{r g}}{2} \rho_{g g}+i \frac{\Omega_{g r}^{*}}{2} \rho_{r r}, \\
\dot{P}_{m}=\Gamma_{m r} \rho_{r r}
\end{gathered}
$$

where $\rho_{g g}, \rho_{r r}$, and $\rho_{r g}$ are the matrix elements of the density operator for the ground state $(g)$ and the selected isolated resonance state $(r)$, and $P_{m}$ describes the population of the MS states $(m)$. The generalized detuning is written as $\Delta=2 \omega-E_{r}+E_{g}-S_{r g}$, where the energy of the resonance state $E_{r}$ includes the shift due to the coupling with the corresponding continuum, and $S_{r g}=S_{r}-S_{g}$ is the laser induced shift. The two-photon Rabi frequencies, $\Omega_{g r}$ and $\Omega_{r g}$, are proportional to the square of the electric field amplitude $\mathcal{E}_{0}^{2}=8 \pi \alpha I$ and have been obtained by eliminating the intermediate ${ }^{1} P^{o}$ bound and continuum states [47]. The single-photon ionization is by far the dominant mechanism for the depletion of the ground state so that $\Gamma_{g} \approx \sigma_{g} I / \omega$, where $\sigma_{g} \approx 5 \mathrm{Mb}$ is the ionization cross section and $I(t)=$ $I_{0} \exp \left[-4(\ln 2) t^{2} / \tau^{2}\right]$ describes the temporal intensity profile of the laser pulse with duration $\tau$ and peak intensity $I_{0}$. The total decay rate of the resonance state is $\Gamma_{r} \approx$ $\Gamma_{r}^{a}+\Gamma_{r}^{f}+\sigma_{r} I / \omega$, where $\Gamma_{r}^{a}$ is the autoionization width, $\Gamma_{r}^{f}$ the total fluorescence width, and $\sigma_{r}$ denotes the photoionization cross section of the atom in the resonance state. The decay rate $\Gamma_{m r}$ of the resonance state to the MS states is calculated as

$$
\Gamma_{m r}=\sum_{\mathfrak{n}} \sum_{m, \boldsymbol{\nu}} \frac{\Gamma_{m \nu_{\mathfrak{n}}}^{f}}{\Gamma_{\nu_{\mathfrak{n}}}} \cdots \frac{\Gamma_{\nu_{2} \nu_{1}}^{f}}{\Gamma_{\nu_{1}}} \Gamma_{\nu_{1} r}^{f} .
$$

Here, $\mathfrak{n}$ is the number of emission steps in a cascade. The inner sum runs over the accessible intermediate states $\left|\nu_{1}\right\rangle, \ldots,\left|\nu_{\mathfrak{n}}\right\rangle$ and the MS states $|m\rangle$, and $\Gamma_{\beta \alpha}^{f}$ and $\Gamma_{\alpha}$ are the partial fluorescence and the total decay widths, respectively. The parameters used in the calculation are provided as Supplemental Material [46]. Decay branching ratios to the MS states vary nontrivially from one resonance to the next and substantially affect the calculated MS yield. A precise treatment of the strong mixing among the singlet and triplet 1 sn $\ell(\ell \geq 3)$ states [48] is required to obtain accurate rates $\Gamma_{m r}$ of the ${ }^{1} D^{e}$ resonances. Our model relies on the branching ratios $\Gamma_{m r} / \Gamma_{r}$, which reproduce the MS yields of isolated 1 sn $\ell$ manifolds in a dc electric field with high accuracy [49].

In Fig. 3(a), the calculated metastable yield $N_{m}(\omega)=$ $P_{m}(t \rightarrow \infty ; \omega)$ is plotted together with the measured yield averaged over the range of pulse energies. The comparison is quite robust since for laser intensities $I_{0} \lesssim 10^{14} \mathrm{~W} / \mathrm{cm}^{2}$, the calculated peak ratios are practically independent of $I_{0}$. There is also no discernible effect due to absorption of an additional photon by the resonance state which would selectively broaden the peaks and reduce the MS yield at higher intensities. To match the measured linewidths with $\tau$ fixed to $100 \mathrm{fs}$ (equal to the full width at half maximum of the Fourier transform of $S_{\lambda}$ ), an additional relaxation rate 
$\gamma_{L}=16 \mathrm{meV}$ of the atomic coherence (e.g., see Ref. [50]) has been introduced in Eq. (1c). The observed width of the two-photon signal is $25 \mathrm{meV}$ at $\hbar \omega=31 \mathrm{eV}$ [inset of Fig. 3(a)], which is narrower than the corresponding onephoton energy resolution $(33 \mathrm{meV})$. This is a clear signature of two-photon absorption where the line shape of the narrow resonance $\left(2 b^{1} S^{e}\right)$ is given by an autoconvolution $(S * S)_{2 \lambda}$ of the single photon spectrum. As seen in Fig. 3(a), the experimental uncertainties were small enough to allow for a reliable identification of the DES on the basis of their calculated energies and the MS yield ratios.

Good agreement with the experimental data in Fig. 3(a) validates the theoretical results. This translates to the yet untested excitation step of the model which may be related to the two-photon absorption cross section. Figure 3(b) presents the two-photon ionization cross section $\sigma^{(2)}$ reconstructed from the parameters calculated by Sánchez et al. [25], which practically coincides with the two-photon absorption cross section because of the relatively small fluorescence decay branching ratios $\Gamma_{r}^{f} / \Gamma_{r}$, and agrees well with our model results [46]. In general, there is a notable difference with respect to the known behavior of the singlephoton absorption cross section with a predominant contribution of the $a^{1} P^{o}$ series below the $N=2$ threshold [51]. According to the propensity rule for dipole matrix elements [52], the Stark mixing preferentially couples DES with matching correlation types, so that the $a$-type DES dominate the single-photon ionization spectrum of $\mathrm{He}$ in a dc electric field environment [53]. The two-photon absorption amplitude involves a product of two dipole matrix elements too, but here the DES are coupled to the singly excited 1 sn $p$ states and to the 1 sep continuum with approximately the same strength, so that different types of DES may exhibit comparable cross sections. In this respect, the situation here is closer to the resonant single-photon absorption, which proceeds from the singly excited $1 s 2 s{ }^{1} S^{e}$ state where both the $a^{1} P^{o}$ and $b{ }^{1} P^{o}$ series dominate the photoelectron spectrum [54].

For both clearly observed resonances $2 b^{1} S^{e}$ and $3 b^{1} D^{e}$ the MS yield is found to saturate [Fig. 4(a)]. The saturation occurs because all the ground state atoms in the focus are ionized before the pulse has reached its global peak intensity $I_{f}$, and subsequent resonant excitation may occur only further away from the focus, where the intensity is lower. The observed trends are reproduced assuming a boxtype laser intensity profile with $20-30 \mu \mathrm{m}$ cutoff diameter [46]. As seen in Fig. 2, a small energy shift of the $3 b^{1} D^{e}$ spectral line is observed, whose origin is attributed to the ac Stark shift. The observed and calculated resonance energy shifts $\left(\sim 13 \mathrm{meV}\right.$ at $\left.I_{f}=10^{14} \mathrm{~W} / \mathrm{cm}^{2}\right)$ are close to the ponderomotive shift $U_{p}=2 \pi \alpha I_{f} / \omega^{2}$, indicating that the electrons in the low-lying doubly excited states experience a similar shift to that of a free electron in the same laser field [Fig. 4(b)]. In examined intensity range the laser field induced coupling of the neighboring resonances at $\sim 2 \omega$ is
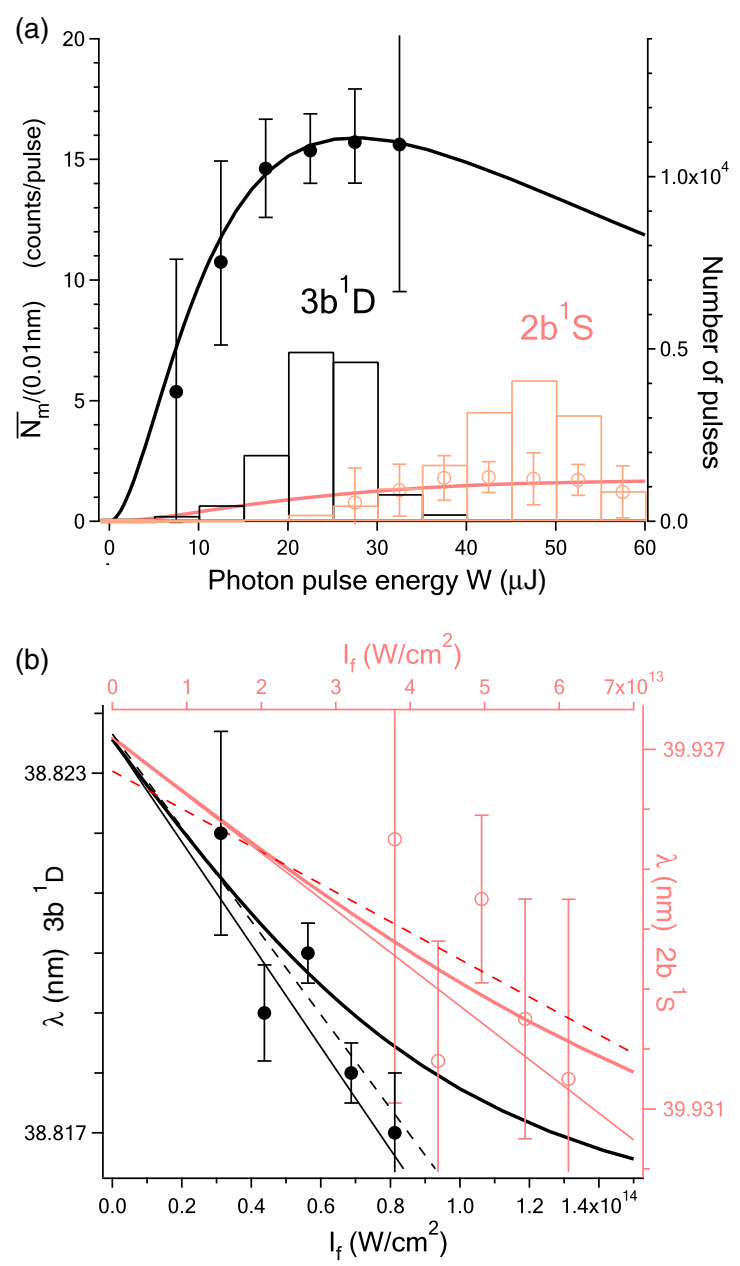

FIG. 4 (color online). (a) Integrated metastable yield versus pulse energy: experimental data (circles), simulated data (curves) [46], and pulse energy distribution (histogram). (b) Resonance energy shift versus pulse intensity: data for $3 b^{1} D^{e}$ (full black circles) and $2 b{ }^{1} S^{e}$ resonance [empty gray (red) circles], linear fit to the experimental data (dashed lines), calculated energy shift $S_{r g} / 2$ (thick solid curve), and the ponderomotive shift divided by 2 (thin solid lines).

negligible because it proceeds through the distant manifold of (continuum) states at energy $\sim \omega$, and the field presence causes only a small shift of resonance energies.

In conclusion, we have revealed hitherto unobserved one-color two-photon above-threshold resonances of isolated He doubly excited states. The spectral features recorded at laser peak intensities as high as $10^{14} \mathrm{~W} / \mathrm{cm}^{2}$ are well reproduced by an effective two-level atom model taking into account a dominant one-photon ionization channel and weak population transfer to the metastable states. Combined with reliable values of zero-field resonance decay parameters, these results validate the calculated two-photon ionization cross section in the region of low-lying doubly excited states of He. The background-free detection of helium metastable atoms coupled with the seeded FEL sources enables high resolution multiphoton 
spectroscopy in the EUV energy range, including large sensitivity to the triplet DES which renders the method suitable for studying the spin-orbit coupling of higher angular momentum states [32]. The same approach can be applied to other atomic and molecular species with more complicated and less studied decay dynamics (neutral dissociation) and to higher laser intensities.

This work was supported by the P1-0112 research program of the Slovenian Research Agency. The research leading to these results has received funding from the European Community's Seventh Framework Programme (FP7/2007-2013) under grant Agreement No. 312284. M. S., L. J., and J.L. acknowledge support from the CNRS through the PEPS SASELEX. M.M. and T.M. acknowledge financial support from SFB 925 at the University of Hamburg. R.F. thanks the Swedish Research Council (VR) for financial support. M. C., C. G., and C. G. acknowledge support by the Italian Slovenian Crossborder Cooperation Programme (CITIUS project).

[1] Y. Nabekawa, H. Hasegawa, E. J. Takahashi, and K. Midorikawa, Phys. Rev. Lett. 94, 043001 (2005).

[2] M. Swoboda, T. Fordell, K. Klünder, J. M. Dahlström, M. Miranda, C. Buth, K. J. Schafer, J. Mauritsson, A. L'Huillier, and M. Gisselbrecht, Phys. Rev. Lett. 104, 103003 (2010).

[3] W. Ackermann et al., Nat. Photonics 1, 336 (2007).

[4] T. Shintake et al., Nat. Photonics 2, 555 (2008).

[5] P. Emma et al., Nat. Photonics 4, 641 (2010).

[6] T. Ishikawa et al., Nat. Photonics 6, 540 (2012).

[7] E. Allaria et al., Nat. Photonics 6, 699 (2012).

[8] C. D. Lin, Phys. Rev. A 29, 1019 (1984).

[9] S. Mannervik, R. Short, D. Sonnek, E. Träbert, G. Möller, V. Lodwig, P. Heckmann, J. Blanke, and K. Brand, Phys. Rev. A 39, 3964 (1989).

[10] M. Stener, P. Decleva, and A. Lisini, J. Phys. B 28, 4973 (1995).

[11] I. Sánchez and F. Martín, J. Chem. Phys. 106, 7720 (1997).

[12] Y. Hatano, Phys. Rep. 313, 109 (1999).

[13] R. Flammini, E. Fainelli, and L. Avaldi, J. Phys. B 33, 1507 (2000).

[14] T. Odagiri, H. Miyagi, M. Murata, H. Fukuzawa, M. Kurokawa, M. Kitajima, and N. Kouchi, J. Phys. B 42, 055101 (2009).

[15] R. P. Madden and K. Codling, Phys. Rev. Lett. 10, 516 (1963).

[16] R. P. Madden, D. L. Ederer, and K. Codling, Phys. Rev. 177, 136 (1969).

[17] V. Schmidt, Rep. Prog. Phys. 55, 1483 (1992).

[18] V. L. Sukhorukov, I. D. Petrov, Ph. V. Demekhin, H. Schmoranzer, S. Mickat, S. Kammer, K.-H. Schartner, S. Klumpp, L. Werner, and A. Ehresmann, J. Phys. B 40, 1295 (2007).
[19] B. Krässig, E. P. Kanter, S. H. Southworth, R. Guillemin, O. Hemmers, D. W. Lindle, R. Wehlitz, and N. L. S. Martin, Phys. Rev. Lett. 88, 203002 (2002).

[20] K. Prince, M. Coreno, R. Richter, M. de Simone, V. Feyer, A. Kivimäki, A. Mihelič, and M. Žitnik, Phys. Rev. Lett. 96, 093001 (2006).

[21] O. Ghafur, W. Siu, P. Johnsson, M. F. Kling, M. Drescher, and M. J. J. Vrakking, Rev. Sci. Instrum. 80, 033110 (2009).

[22] P. Lablanquie et al., Phys. Rev. Lett. 106, 063003 (2011).

[23] Y. Hikosaka, P. Lablanquie, M. Ahmad, F. Penent, J. H. D. Eland, and R. I. Hall, J. Phys. B 37, 283 (2004).

[24] G. Tanner, K. Richter, and J.-M. Rost, Rev. Mod. Phys. 72, 497 (2000).

[25] I. Sánchez, H. Bachau, and E. Cormier, J. Phys. B 28, 2367 (1995).

[26] L. A. A. Nikolopoulos and P. Lambropoulos, J. Phys. B 34, 545 (2001).

[27] A. Palacios, T. N. Rescigno, and C. W. McCurdy, Phys. Rev. A 77, 032716 (2008).

[28] D. R. Herrick and O. Sinanoğlu, Phys. Rev. A 11, 97 (1975).

[29] J.-E. Rubensson, C. Såthe, S. Cramm, B. Kessler, S. Stranges, R. Richter, M. Alagia, and M. Coreno, Phys. Rev. Lett. 83, 947 (1999).

[30] M. K. Odling-Smee, E. Sokell, P. Hammond, and M. A. MacDonald, Phys. Rev. Lett. 84, 2598 (2000).

[31] T. Gorczyca, J.-E. Rubensson, C. Såthe, M. Ström, M. Agåker, D. Ding, S. Stranges, R. Richter, and M. Alagia, Phys. Rev. Lett. 85, 1202 (2000).

[32] F. Penent, P. Lablanquie, R. Hall, M. Žitnik, K. Bučar, S. Stranges, R. Richter, M. Alagia, P. Hammond, and J. Lambourne, Phys. Rev. Lett. 86, 2758 (2001).

[33] J. Lambourne et al., Phys. Rev. Lett. 90, 153004 (2003).

[34] J.-E. Rubensson, A. Moise, A. Mihelič, K. Bučar, M. Žitnik, and R. Richter, Phys. Rev. A 81, 062510 (2010).

[35] M. Žitnik, F. Penent, P. Lablanquie, A. Mihelič, K. Bučar, R. Richter, M. Alagia, and S. Stranges, Phys. Rev. A 74, 051404 (2006).

[36] A. Mihelič and M. Žitnik, Phys. Rev. Lett. 98, 243002 (2007).

[37] M. Ström, C. Såthe, M. Agåker, J. Söderström, J.-E. Rubensson, S. Stranges, R. Richter, M. Alagia, T. Gorczyca, and F. Robicheaux, Phys. Rev. Lett. 97, 253002 (2006).

[38] T. Weber et al., Opt. Express 8, 368 (2001).

[39] H. Hasegawa, E. J. Takahashi, Y. Nabekawa, K. L. Ishikawa, and K. Midorikawa, Phys. Rev. A 71, 023407 (2005).

[40] M. Kurka et al., New J. Phys. 12, 073035 (2010).

[41] A. Hishikawa et al., Phys. Rev. Lett. 107, 243003 (2011).

[42] P. O'Keeffe, A. Mihelič, P. Bolognesi, M. Žitnik, A. Moise, R. Richter, and L. Avaldi, New J. Phys. 15, 013023 (2013).

[43] T. Mazza et al., Nat. Commun. 5, 3648 (2014).

[44] M. Zangrando et al., Rev. Sci. Instrum. 80, 113110 (2009).

[45] V. Lyamayev et al., J. Phys. B 46, 164007 (2013).

[46] See Supplemental Material at http://link.aps.org/ supplemental/10.1103/PhysRevLett.113.193201 for more 
details about experimental conditions and data analysis and to access calculated model parameters for low lying ${ }^{1} S^{e}$ and ${ }^{1} D^{e}$ resonances in He.

[47] P. Lambropoulos and P. Zoller, Phys. Rev. A 24, 379 (1981).

[48] G. W. F. Drake, Handbook of Atomic, Molecular, and Optical Physics, edited by G. W. F. Drake (Springer, New York, 2006).

[49] A. Mihelič, M. Žitnik, K. Bučar, L. Avaldi, and R. Richter, Phys. Rev. A 89, 063422 (2014).
[50] A. T. Georges and P. Lambropoulos, Phys. Rev. A 18, 587 (1978).

[51] M. Domke, K. Schulz, G. Remmers, G. Kaindl, and D. Wintgen, Phys. Rev. A 53, 1424 (1996).

[52] X. M. Tong and C. D. Lin, Phys. Rev. Lett. 92, 223003 (2004).

[53] J. Harries, J. Sullivan, J. Sternberg, S. Obara, T. Suzuki, P. Hammond, J. Bozek, N. Berrah, M. Halka, and Y. Azuma, Phys. Rev. Lett. 90, 133002 (2003).

[54] M. Alagia et al., Phys. Rev. Lett. 102, 153001 (2009). 